\title{
Overhead Spine Arch Analysis of Dairy Cows from Three-Dimensional Video
}

\author{
K. ABDUL JABBAR, M. F. Hansen, M. L. Smith, and L. N. Smith \\ Centre for Machine Vision, Bristol Robotics Laboratory \\ University of the West of England, Bristol, United Kingdom
}

\begin{abstract}
We present a spine arch analysis method in dairy cows using overhead 3D video data. This method is aimed for early stage lameness detection. That is important in order to allow early treatment; and thus, reduce the animal suffering and minimize the high forecasted financial losses, caused by lameness. Our physical data collection setup is non-intrusive, covert and designed to allow full automation; therefore, it could be implemented on a large scale or daily basis with high accuracy. We track the animal's spine using shape index and curvedness measure from the 3D surface as she walks freely under the 3D camera. Our spinal analysis focuses on the thoracic vertebrae region, where we found most of the arching caused by lameness. A cubic polynomial is fitted to analyze the arch and estimate the locomotion soundness. We have found more accurate results by eliminating the regular neck/head movements' effect from the arch. Using 22-cow data set, we are able to achieve an early stage lameness detection accuracy of $95.4 \%$.
\end{abstract}

Keywords: 3D vision, curvature, dairy cow, early lameness, lameness detection, spine arch, vertebrate

\section{INTRODUCTION}

Lameness in dairy cows is acknowledged as a serious problem that affects an animal's welfare and thus, productivity $[1,2]$. Subsequently, lameness account for tremendous financial losses in extensively managed dairy farms [2]. Regardless of causes, early detection and prompt treatment minimizes losses and reduces animal suffering [3]. We therefore present a sensitive analysis that aims to establish a reliable threshold for early stage lameness. Because of the animals' natural resistance to pain caused by lameness; they tend to shift their body weight towards the contralateral limb [4]. This subsequently increases the spine's arch. Hence, the spine posture is considered as a reliable measure to predict if the cow is showing signs of discomfort, and subsequently is lame [1].

The majority of previous vision-based research is based on estimating the back's curvature to predict gait soundness. However, automated methods such as $[5,6]$ present a coarse-grained level for the ground truth locomotion/lameness score (LS). Hence, it is not possible to ascertain the sensitivity towards minor deviations from healthy gaits in above state-of-the-art methods. On the other hand, in order to obtain an early stage lameness detection, our study is focused to investigate the small sensitive region, i.e. from LS 1 to LS 2 in a 5-level LS system. We have developed a different approach as compared to both $[5,6]$ by fitting a cubic polynomial on the extracted spine region from the 3D depth map. We also detect the neck area in order to eliminate neck movements' effect on the back arch. The back arch is then estimated using the curvature of the fitted polynomial.

\section{DATA}

Robust spine tracking or extracting is an essential part of this study. Therefore, a traditional 2D imagery is not suitable as it presents many problems (e.g. segmentation of the foreground from the background, occlusions). On the other hand, 3D data provides the surface topology information, which is critically used to apply an angular measure for a robust spine extraction. Furthermore, assessing the locomotion of the animal requires observing how it walks. This takes place over time, and therefore a 4D (3D video) analysis lends itself well. Our overhead capturing setup (i.e. on top of the herd) is mainly put together because of the general feasibility it offers for automation. An overhead setup enables a completely covert and non-intrusive option; this directly affects the accuracy as we keep human involvement to the minimum. It further provides a hardware configuration which is less prone to damage and the presence of noisy image backgrounds.

We present a preliminary data set of 22 Holstein Friesian dairy cows collected at the Bridge Farm, Glastonbury, United Kingdom. A custom race has been built next to the milking parlor which forces the cows to walk unconstrained in single file underneath a standard depth-sensor camera. The camera is installed at a height of $3.69 \mathrm{~m}$ off the floor. At this 
height, each cow's locomotion provides at least two full gait cycles (8 foot falls). An experienced local observer has scored each cow using the Sprecher et al [7] LS system. Because we are investigating a close/sensitive LS region, only cows that repeatedly received manual LS of either 1,2 or 3 across three sessions were used.

\section{PRE-PROCESSING AND FEATURES}

\subsection{Pre-processing}

First, the background (an image of the cow race when there is no cow present) is subtracted and a height threshold is applied to eliminate surrounding object pixels. The extraneous information (noise due to camera height) in the resulting image are filtered-out. A symmetric Gaussian low-pass filter is used to remove the quantization artifacts from the raw
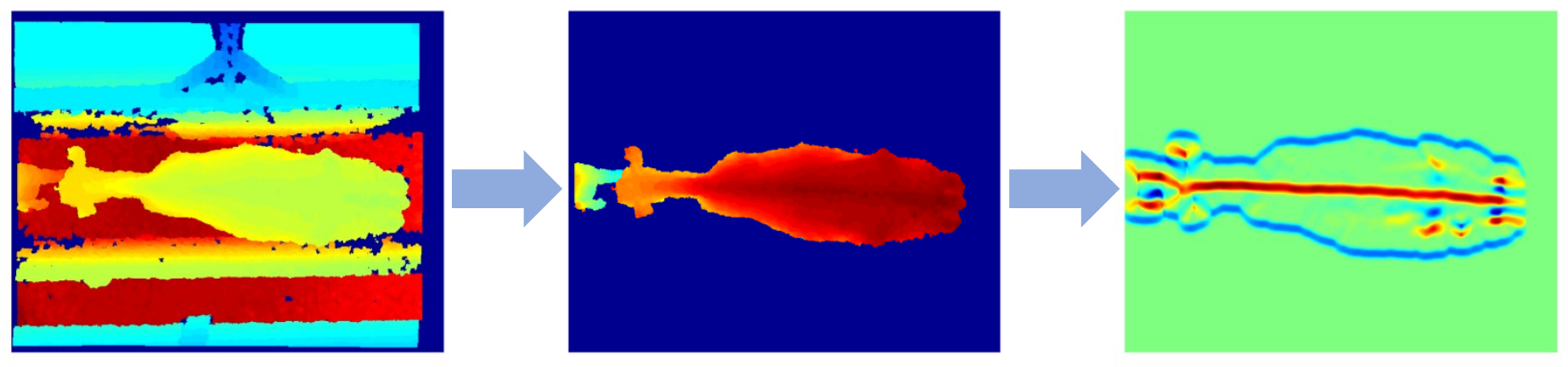

image.

Figure 1. A simplified pipeline for pre-processing and features extraction. The first image (from the left) is a raw depth image from the camera in the race; followed by the same image with the background removed, height threshold applied and smoothed to prevent limiting any curvature information; followed by a binary converted image of the curvedness threshold to track the spine. Notive how the distinctly curved features (highest convex regions i.e. spine, hooks and pins) are clearly visible.

\subsection{Automated Spine Features}

We extract the spine from the processed 3D surface using a high curvedness (convex) measure and the shape index, as defined by Koenderink and van Doorn [8]:

$$
S=\frac{2}{\pi} * \arctan \left(\frac{\kappa_{1(x, y)}+\kappa_{2(x, y)}}{\kappa_{1(x, y)^{-\kappa_{2}}(x, y)}}\right)
$$

Where $s$ is the shape index, it represents the overall grade for a given shape in the surface (e.g. $(-1<s<-1 / 2)$ for concave and $(1 / 2<s<1)$ for convex shapes). The positive principle curvatures $\left(\kappa_{1}, \kappa_{2}\right)$ are used in the convex range in our case.

$\bar{C}$, the curvedness measure of the 3D shape is calculated by (4) and it represents the normalized magnitude of the combined principle curvatures. This measure tells how curved a point in the surface is. The principal curvatures are calculated from the Gaussian and mean curvatures of the surface. They correspond to the orthogonal axes which reflect a point on the object's surface.

$$
\begin{aligned}
& C_{(x, y)}=\sqrt{\frac{\kappa_{1}^{2}(x, y)+\kappa_{2}^{2}(x, y)}{2}} \\
& C_{\text {max }}=\max (C) \\
& \bar{C}_{(x, y)}=\frac{C_{(x, y)}}{C_{\max }}
\end{aligned}
$$

By thresholding the curvedness, the most prominent convex features (which corresponds to peaks) are visible - as shown in Figure 1. The spine represents the largest connected object given in a binary converted image of the curvedness 
threshold. Figure 1 illustrates a simplified image processing pipeline, as described above. This process is repeated for each frame in the data.

\subsection{Automatic Neck Detection}

Neck activity or head bob measure from sensor data or human observation is an investigated variable that correlates to lameness [9]. Lame cows tend to use their head to shift the weight forward (counterbalance), in case the affected limb is at the rear. This subsequently affects the spine's curvedness measure. However, in our case, as we are analyzing the spine arch a single lameness trait, the neck area is eliminated to limit any additional curvedness to the arch. We convert the $3 \mathrm{D}$ surface to a flat, disk-shaped morphological structuring element with a radius of 10 . This $2 \mathrm{D}$ neighborhood is used for a morphological close operation (dilation then erosion). The resulted image (as shown in Figure 2), is used to locate the neck region, which is restricted by $n=s<70 \& s>50$, where $s$ is the number of pixels in the neck region $n$. From the last element in $n$, we check whether we have 10 consecutive preceding $s$ values that are restricted for the $n$
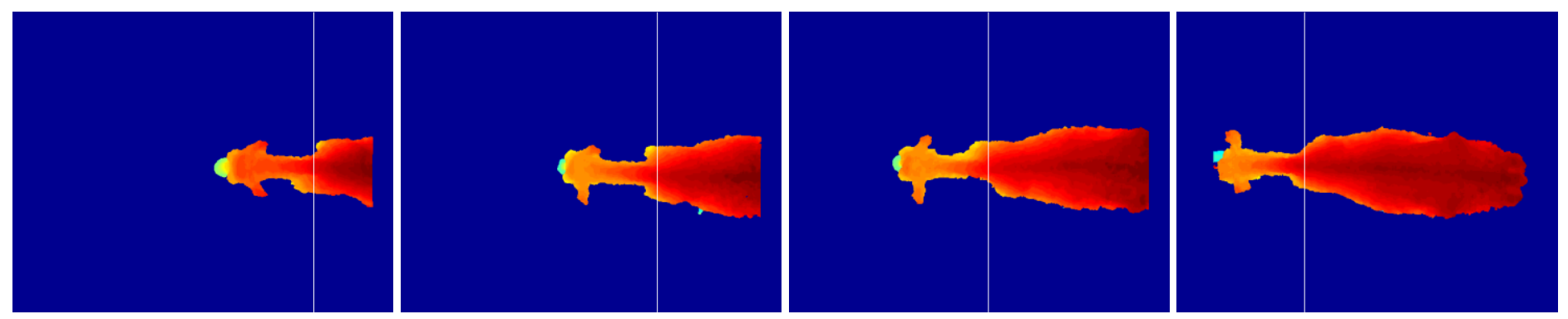

region.

Figure 2. Top row shows a binary image that is used for locating the neck region. The heighligted neck (cervical verterbrae) region in this example is 72-pixels long. The Bottom row is an example of robust neck tracking progress for the same cow (starting from left to right). Each image is taken after 13 frames, the camera opertaes at 30 frames/second.

\section{SPINE ARCH ANALYSIS AND RESULTS}

\subsection{Spine Extraction and Polynomial Fitting}

The extracted spine represents the most convex region from the 3D surface. We have found a fitted cubic polynomial through the highest convex points in the spine region a suitable technique to analyze the arch. The spine length is restricted in this analysis to cover the thoracic vertebrae region, where most of the spinal arching occurs. This means that we discard the first few frames from the analysis where we don't have the full thoracic vertebrae region (e.g. first left image in the bottom row in Figure 2). The same applies when the majority of the spinal region in the frame is caudal vertebrae, i.e. towards the end of the locomotion. We further mitigate the caudal vertebrae arching effect (mainly caused by the tail movements) by eliminating a small 50-pixels region from the end of the spine. Figure 3 demonstrates the automated spine extraction, neck detection and polynomial fitting in a single frame from the processed 3D data of a lame and a healthy cow. For an increased sensitivity towards arching, we assess the locomotion using the normalized minimum B-term of the fitted polynomial, across the entire locomotion frames.

\subsection{Results}

On a 22-cow data set -as shown in Figure 4-, we are able to establish a threshold between LS 1 and 2. The spinal arching correlates well with the manual LS. Thus, serving the main purpose of this analysis as we are more interested in 
the sensitive early stage lameness rather than the individual classifications of LS 3, 4 and 5. All lame cows in the presented data (including cows in LS 2, i.e. in a mildly lame condition) are identified within the early lameness
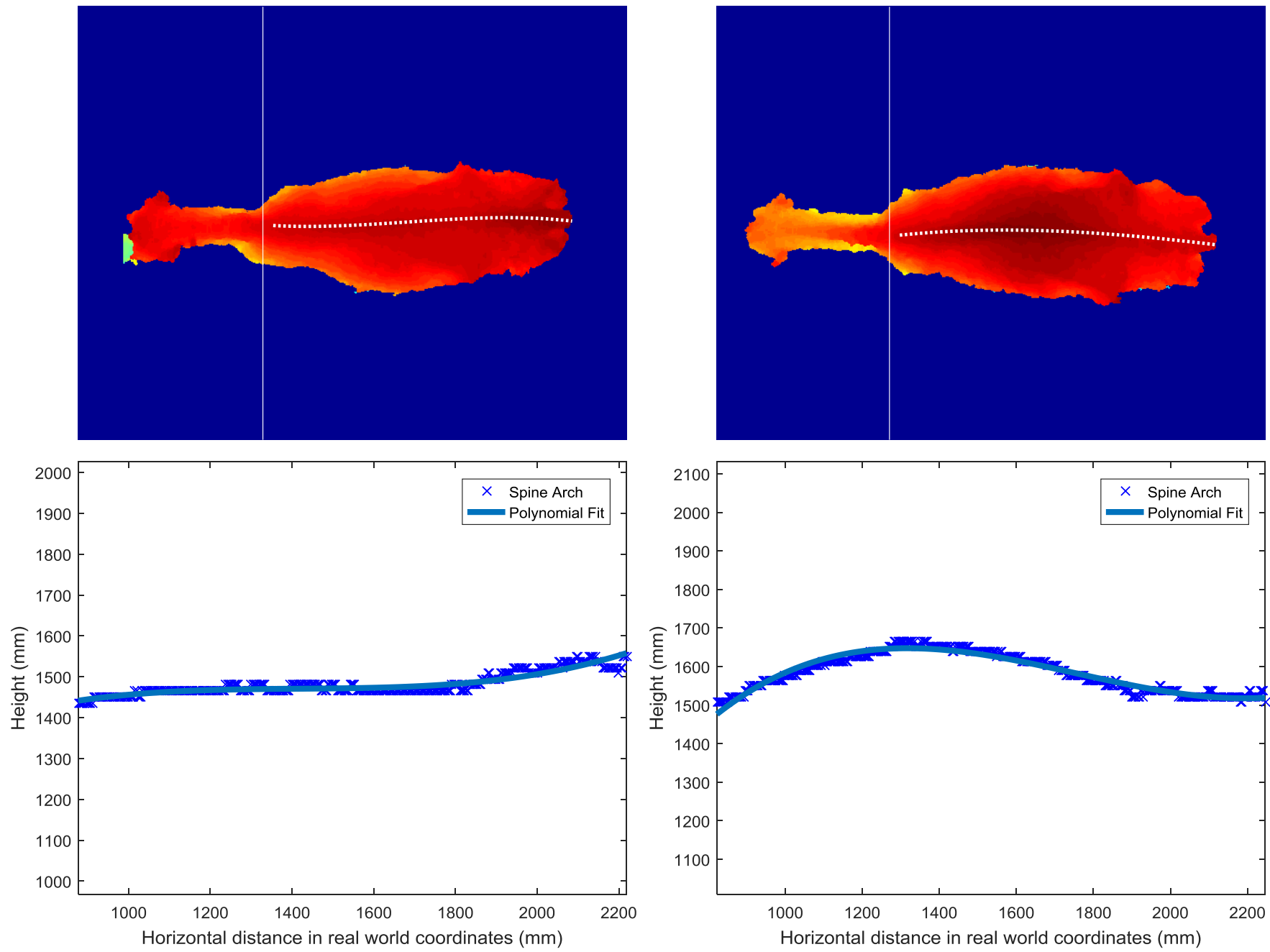

threshold. Thus, we achieve an accuracy of $95.4 \%$ using a binary classification between LS 1 and LS 2, 3, 4 and 5 combined. However, we have an exception for a healthy cow (LS 1) receiving a high spine arching value. This individual cow (highlighted in the red box in Figure 4) showed an unusual and regular head bob while walking, which resulted in high B-terms.

Figure 3. Back arching examples for a lame cow (right coloumn) and a healthy cow (left coloumn). Top row shows both processed cows from a topdown view, with the spine features extracted and the neck area identified. Bottom row shows the back arch in each cow from a side view, with its polynomial fit. Notice the clear arch in the lame cow.

\section{CONCLUSION}


The presented 3D overhead data is a suitable approach given the nature of the application, and the type of convex features required. Thus, the system could be implemented on daily basis to allow herdsmen detect small changes, and

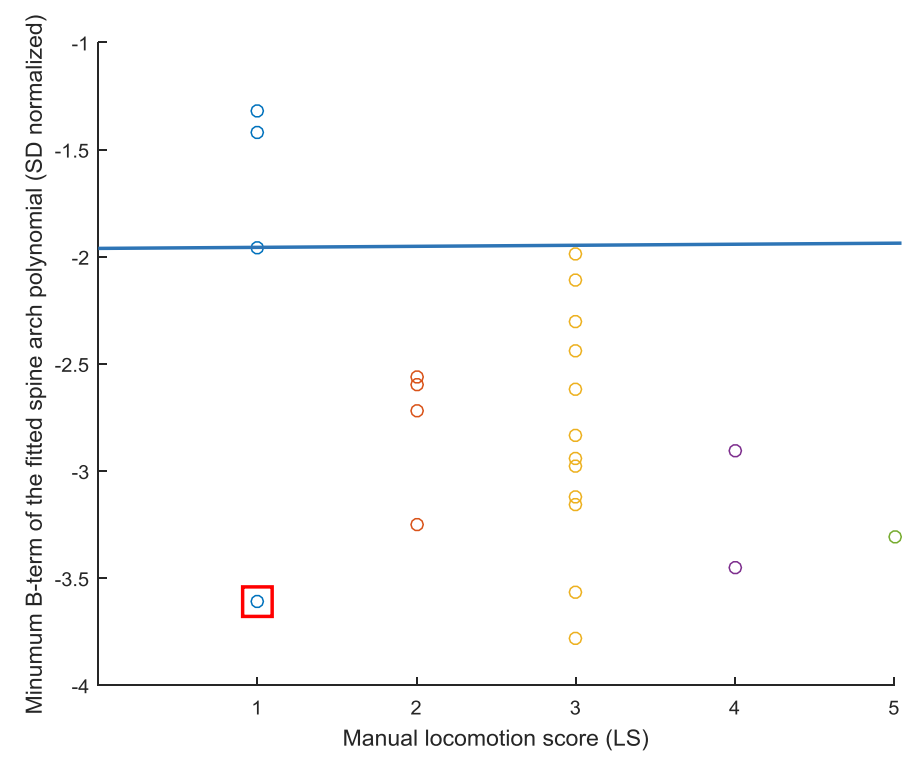

act accordingly. We are also able to robustly track the spine and the neck. We have found the spinal arching in the thoracic vertebrae region a sensitive lameness indicator. Future work will focus on developing a comprehensive assessment by combining other locomotion traits (e.g. head movements, gait asymmetry).

Figure 4. Early stage lameness detection results from the spine arch. All 22 cows are manually scored using Sprecher et al [7] LS system - where LS 1 is healthy and LS 5 is severly lame. The minumum B-term represnts the spinal arching from the fitted cubic polynomial. From this data-set, an early stage lameness threshold is identified at -1.95 .

\section{ACKNOWLEDGMENTS}

This research is funded by the University of the West of England (Bristol, UK). Special thanks to the observer, E. F. Wright and the staff of Kingshay Farming and Conservation Ltd. and Bridge Farm (Glastonbury, UK); for their extensive help and input during data collection at the farm.

\section{REFERENCES}

[1] A. Schlageter-Tello, E. Bokkers, P. Groot Koerkamp, T. Van Hertem, S. Viazzi, C. Romanini, I. Halachmi, C. Bahr, D. Berckmans and K. Lokhorst, "Relation between observed locomotion traits and locomotion score in dairy cows", J. of Dairy Sci., 98, 8623-8633, (2015).

[2] J. A. Willshire and N. J. Bell, “An economic review of cattle lameness," Catt. Prac., 17, 136-141, (2009).

[3] K. A. Leach, D. A. Tisdall, N. J. Bell, D. C. J. Main, and L. E. Green, "The effects of early treatment for hindlimb lameness in dairy cows on four commercial UK farms," The Vet. J., 193, 626-632, (2012).

[4] S. Neveux, D. Weary, J. Rushen, M. von Keyserlingk and A. de Passillé, "Hoof Discomfort Changes How Dairy Cattle Distribute Their Body Weight", J. of Dairy Sci., 89, 2503-2509, (2006).

[5] S. Viazzi, C. Bahr, T. Van Hertem, A. Schlageter-Tello, C. Romanini, I. Halachmi, C. Lokhorst and D. Berckmans, "Comparison of a three-dimensional and two-dimensional camera system for automated measurement of back posture in dairy cows", Comput. and Electronics in Agri., 100, 139-147, (2014).

[6] T. Van Hertem, S. Viazzi, M. Steensels, E. Maltz, A. Antler, V. Alchanatis, A. Schlageter-Tello, K. Lokhorst, E. Romanini, C. Bahr, D. Berckmans and I. Halachmi, "Automatic lameness detection based on consecutive 3D-video recordings", Bioseys. Eng., 119, 108-116, (2014).

[7] D. J. Sprecher, D. E. Hostetler, and J. B. Kaneene, “A lameness scoring system that uses posture and gait to predict dairy cattle reproductive performance," Theriogenology, 47, 1179-1187, (1997). 
[8] J. J. Koenderink and A. J. van Doorn, "Surface shape and curvature scales," Image and Vision Comput., 10, $557-$ $564,(1992)$.

[9] T. Van Hertem, E. Maltz, A. Antler, C. E. B. Romanini, S. Viazzi, C. Bahr, A. Schlageter-Tello, C. Lokhorst, D. Berckmans, and I. Halachmi, "Lameness detection based on multivariate continuous sensing of milk yield, rumination, and neck activity," J. of Dairy Sci., 96, 4286-4298, (2013). 